\title{
Fatores associados ao estado nutricional de iodo no grupo materno-infantil: uma revisão sistemática
}

\author{
Factors associated with the nutritional status of iodine \\ in the maternal-infant group: a systematic review
}

Aline Carare Candido (https://orcid.org/0000-0002-6259-4786) ${ }^{1}$

Silvia Eloíza Priore (https://orcid.org/0000-0003-0656-1485) ${ }^{1}$

Mariana de Souza Macedo (https://orcid.org/0000-0003-4564-6334) ${ }^{1}$

Sylvia do Carmo Castro Franceschini (https://orcid.org/0000-0001-7934-4858) ${ }^{1}$

${ }^{1}$ Departamento de Nutrição e Saúde, Universidade Federal de Viçosa. Avenida Peter Henry Rolfs $s / n^{\circ}$, Campus Universitário. 36570-900 Viçosa MG Brasil.

alinecarrare@gmail.com
Abstract This article sets out to identify the socio-demographic and nutritional factors associated with the nutritional status of iodine in the maternal-infant group. The review was based on the Prisma method and the Medline (PubMed), Scopus and Lilacs databases were consulted. The search was conducted in December 2018, using the key words nutritional status, iodine, infant, pregnant and lactating. The selection followed the stages of excluding duplicated articles, reading the titles, abstracts and texts in full. A total of 1,500 studies were identified and 26 met the inclusion criteria. The methodological quality of the studies was evaluated using the questionnaire proposed by Downs and Black. There was an association between the sociodemographic factors of income, education, maternal and gestational age, uniformity and place of residence in rural or urban areas with the nutritional state of iodine. In relation to dietary factors, the consumption of supplements, iodized salt and dietary sources of iodine, especially dairy products, play a protective role to avoid the deficiency. It is necessary to create more specific and effective strategies that address all the factors that affect the nutritional state of iodine to avoid the disorders resulting from the deficiency. Key words Iodine, Nutritional status, Maternal-infant group
Resumo $O$ objetivo deste artigo é identificar os fatores sociodemográficos e alimentares associados ao estado nutricional de iodo do grupo materno-infantil. A revisão foi baseada no método PRISMA. As bases consultadas foram: Medline (PubMed), Scopus e Lilacs. A busca ocorreu em dezembro de 2018, utilizando os descritores estado nutricional, iodo, lactente, gestante e lactante. A seleção seguiu as etapas de exclusão dos duplicados, leitura dos títulos, resumos e análise na integra. Foram identificados 1.500 estudos e 26 contemplaram os critérios de inclusão. A qualidade metodológica dos estudos foi avaliada pelo questionário proposto por Downs e Black. Houve associação entre os fatores sociodemográficos renda, escolaridade, idade materna e gestacional, paridade e local de residência rural ou urbano com o estado nutricional de iodo. Já em relação aos fatores alimentares, o consumo de suplementos, sal iodado e alimentos fontes de iodo, em especial os produtos lácteos, exercem papel protetor contra a deficiência. É preciso criar estratégias mais específicas, efetivas e que contemplem todos esses fatores que afetam o estado nutricional de iodo para evitar as desordens provocadas pela deficiência.

Palavras-chave Iodo, Estado nutricional, Materno-infantil 


\section{Introdução}

O iodo é um micronutriente constituinte dos hormônios tireoidianos triiodotironina (T3) e tiroxina (T4), fundamentais para o desenvolvimento do sistema nervoso central no período embrionário ${ }^{1}$. Ele é encontrado em pequenas quantidades nos alimentos e sua concentração depende de inúmeros fatores, como as condições do solo e geoclimáticas, a irrigação e os fertilizantes utilizados ${ }^{2}$. Os produtos lácteos e os frutos do mar são considerados as principais fontes ${ }^{3}$.

A deficiência de iodo acomete todas as faixas etárias e a Organização Mundial de Saúde a considera como "a causa evitável mais importante de danos cerebrais em lactentes"2. Em gestantes, a deficiência pode provocar aborto espontâneo e nódulos na tireoide e no feto pode causar hipotireoidismo neonatal, cretinismo, retardo no crescimento e no desenvolvimento neuropsicomotor ${ }^{4}$. Já em crianças, as principais consequências identificadas são retardo neurológico, que comina em incremento dos gastos com saúde e educação, gerando prejuízos sociais e econômicos para os países ${ }^{5}$.

A necessidade nutricional de iodo varia em função da idade e do estado fisiológico². Durante a gestação, ocorrem alterações fisiológicas que aumentam a necessidade de iodo, como a estimulação hormonal da tireoide, a transferência de hormônios ao feto e o aumento da filtração glomerular que elimina cerca de $30 \%$ a $50 \%$ de iodo na urina ${ }^{2,5}$.

Além dos fatores alimentares, os sociodemográficos são determinantes para o estado nutricional de iodo, uma vez que renda, educação e condições de moradia podem influenciar sobre a disponibilidade alimentar qualitativa e quantitativa, afetando diretamente a adequação do consumo de micronutrientes ${ }^{1}$.

São poucos os estudos que abordam quais os fatores que podem estar associados ao estado nutricional de iodo de gestantes, nutrizes e lactentes, por isso, o nosso intuito com este artigo foi fazer um compilado da literatura para identificar esses fatores, para, assim, subsidiar a discussão e a elaboração de estratégias mais específicas e efetivas no enfretamento da deficiência de iodo no grupo materno infantil.

\section{Metodologia}

Trata-se de uma revisão sistemática baseada no método PRISMA (Preferred Reporting Items for
Systematic reviews and Meta-Analyses) ${ }^{6}$. A pergunta norteadora foi "quais os fatores que poderiam estar associados ao estado nutricional de iodo no grupo materno-infantil?".

\section{- População de Interesse:}

Para este artigo, optamos em trabalhar com o grupo materno-infantil, uma vez que com a redução da faixa de iodação para 15 a 45 ppm, levando em consideração o consumo diário de $5 \mathrm{~g}$ de sal iodado, a recomendação de ingestão de 250 $\mu \mathrm{g} /$ dia para gestante e nutriz não é alcançada, podendo causar agravos à saúde infantil2 ${ }^{2}$. Logo, esse grupo é mais susceptível à deficiência de iodo, levando em consideração o aumento da necessidade nutricional devido a mudanças fisiológicas e ao fato de que o sal é a principal fonte desse nutriente na alimentação, devido a quantidade traços presente nos demais alimentos. Dessa forma, seria mais viável realizar uma revisão sistemática para identificar os possíveis fatores que podem contribuir para a deficiência de iodo no grupo materno-infantil, devido à sua vulnerabilidade biológica e fisiológica.

- Estratégia de Busca:

As bases de dados consultadas foram: Medline (PubMed - Nacional Library of Medicine and the Nacional Institutos Health), Lilacs (Literatura Latino-Americana e do Caribe em Ciências da Saúde) e Scopus.

Os descritores indexados no sistema de Descritores em Ciência da Saúde (DeCS) foram estado nutricional, iodo, lactente, gestante e lactante. Para a busca foram realizadas três combinações em cada base de dados: ("estado nutricional" de iodo AND gestante); ("estado nutricional" de iodo AND lactente) e ("estado nutricional" de iodo AND lactante). A pesquisa foi realizada com os descritores nos idiomas português, inglês e espanhol.

A busca foi realizada por dois revisores independentes, em dezembro de 2018, sem delimitação de datas, utilizando os filtros humanos e mulheres. Após a pesquisa, foram registrados os dados dos artigos (autores, local, desenho do estudo, público alvo, os fatores sociodemográficos e alimentares) em uma planilha no Excel. Depois, foram eliminadas as duplicidades por base e entre as bases.

\section{- Seleção dos Artigos:}

A seleção ocorreu de maneira independente, por dois pesquisadores. Quando a opinião era distinta em relação à inclusão dos estudos, um terceiro pesquisador era consultado. Primeiro realizamos a leitura dos títulos, depois dos resumos e, por fim, do artigo completo. 
Foram incluídos estudos realizados com crianças de até 6 anos de idade, gestantes, lactantes e que abordaram os fatores sociodemográficos (renda, escolaridade, local de residência, ocupação, idade da mãe e paridade) e o hábito alimentar, publicados nos idiomas português, inglês ou espanhol.

Excluiu-se estudos experimentais, teses, dissertações, revisão bibliográfica e os artigos que não abordaram o tema proposto.

Além das bases de dados consultadas, foi realizada uma busca reversa, no entanto nenhum artigo contemplou os critérios de inclusão.

- Qualidade Metodológica:

A qualidade metodológica e o risco de viés dos estudos foram avaliados pelo questionário proposto por Downs e Black ${ }^{7}$, que contém 27 perguntas divididas em quatro categorias: relato de estudo (principais achados descritos), validade externa (avalia representatividade), validade interna (investiga vieses e fatores de confusão) e poder do estudo. Foram excluídas 10 das 27 perguntas, pois se referiam a estudos experimentais. Cada resposta foi pontuada em "0" (caso não atendesse ao critério avaliado) ou "1" (se o critério fosse atendido), sendo o máximo 17 pontos.

\section{Resultados}

A pesquisa científica resultou em 1.500 estudos. Depois de eliminar as duplicidades por base e entre as bases restaram 460 artigos. Após a leitura dos títulos, resumos e artigos completos, foram selecionados 26 (Figura 1).

Dos estudos incluídos, $61,5 \%$ foram de delineamento transversal, publicados de $2003^{8}$ a $2017^{9,10}$, sendo que $69,2 \%(\mathrm{n}=18)$ realizaram a pesquisa com gestantes, $46,1 \%(\mathrm{n}=12) \mathrm{com}$ crianças e $23,0 \%(n=6)$ com lactantes. O tamanho amostral variou de $30^{7}$ a $655.811^{11}$ (Quadro 1).

$\mathrm{Na}$ avaliação da qualidade metodológica e dos riscos de viés dos artigos, a menor pontuação foi 12 pontos $(3,84 \%)$ e a maior $17(30,76 \%)$. $\mathrm{Na}$ categoria relato de estudo, $19,23 \%(\mathrm{n}=5)$ não descreveram se houve perda no acompanhamento. Na validade externa, $30,76 \%(\mathrm{n}=8)$ dos estudos não afirmaram a representatividade da amostra. Já na validade interna, $3,84 \%(\mathrm{n}=1)$ não reportaram a perda de indivíduos e, por fim, apenas $34,6 \%$ apresentaram o poder estatístico do estudo. Os demais quesitos avaliados obtiveram a pontuação de um, atendendo o critério de qualidade. Dos artigos avaliados, 69,23\% (n
$=18$ ) atingiram pontuação $\geq 15$ pontos, demostrando ótima qualidade metodológica, conferindo segurança para afirmar os achados.

Os estudos incluídos demostraram que os fatores sociodemográficos renda, escolaridade da mãe, idade materna e gestacional, paridade e local de residência rural ou urbano se associaram ao estado nutricional de iodo. Porém, a precariedade de condições básicas de sobrevivência, como saneamento básico, condições do domicílio e ausência de instalações elétricas influenciaram negativamente no estado de iodo do grupo materno-infantil.

Já em relação ao hábito alimentar, o consumo de suplementos, sal iodado e alimentos fontes de iodo, em especial os produtos lácteos, exerceram papel protetor contra a deficiência (Gráfico 1). Porém, verificou-se que o uso de fórmula infantil foi melhor associado ao estado nutricional de iodo quando comparado ao consumo de leite materno.

\section{Discussão}

No mundo, o número de países com deficiência de iodo diminuiu de 54 em 2003 para $32 \mathrm{em}$ $2011^{12}$. No entanto, apesar dos notáveis progressos, 1,88 bilhões de pessoas ainda possuem ingestão insuficiente, sendo que gestantes, nutrizes e lactentes formam o grupo mais vulnerável ${ }^{13}$. A iodação do sal, foi reconhecida como a estratégia mais efetiva e econômica para evitar a deficiência, visto que o sal é consumido diariamente por todas as faixas etárias ${ }^{14}$.

A renda em 50\% ( $n=13)$ dos estudos incluídos se relacionou ao estado nutricional de iodo, onde crianças pertencentes a famílias sem instalações sanitárias e eletricidade; e que seus pais não possuíam vínculo empregatício foram menos propensas a usar sal iodado. Estudo transversal realizado na Índia com 287.247 crianças, cerca de $45,8 \%$, eram de baixa renda e por isso mais propensas $(\mathrm{OR}=2,55)$ a usar sal não ioda$\mathrm{do}^{15}$. Nas gestantes e lactantes observou-se que a baixa renda pode estar diretamente relacionada ao estado nutricional inadequado de iodo ${ }^{16-20}$. Esses dados são alarmantes, uma vez que a menor renda, na maioria das vezes, está associada a menor nível de instrução, o que pode estar levando à redução do consumo de sal iodado, o que afeta diretamente o estado nutricional iódico.

A maior escolaridade foi fator protetor contra a deficiência de iodo em $46,1 \%(\mathrm{n}=12)$ dos estudos. Na Etiópia, estudo longitudinal eviden- 


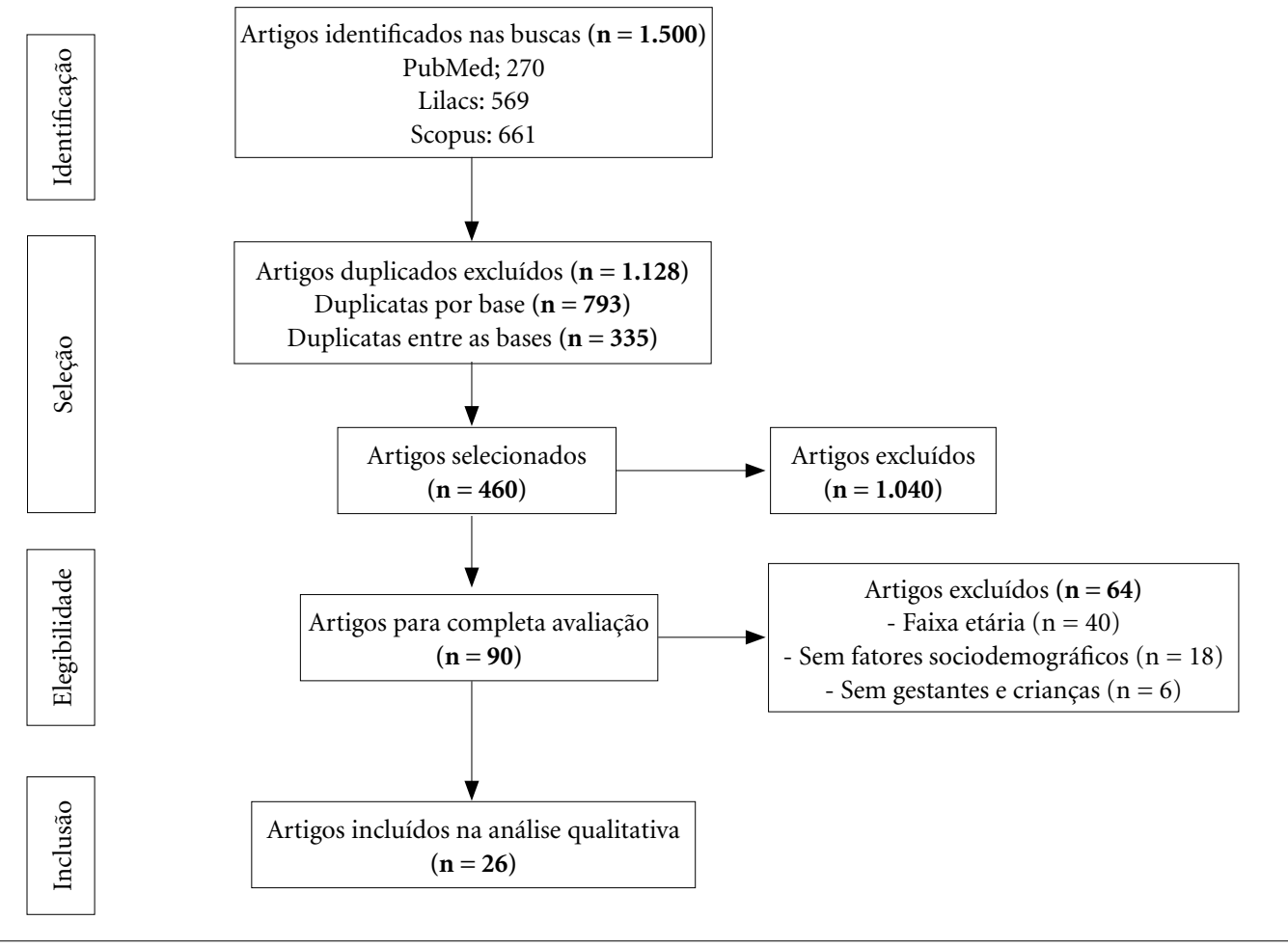

Figura 1. Fluxograma do processo de identificação e seleção dos artigos incluídos. Fonte: PRISMA ${ }^{6}$.

ciou que as gestantes analfabetas e pertencentes a famílias com 5 membros ou mais tinham maior risco de apresentar deficiência de iodo ${ }^{21}$. $\mathrm{Na}$ Índia, uma pesquisa verificou correlação negativa entre a escolaridade materna e a iodúria ${ }^{22}$.

A idade materna é outro fator que se associa ao estado nutricional de iodo. No Reino Unido, estudo transversal realizado com gestantes detectou correlação negativa entre idade materna e iodúria ${ }^{23}$. Já na Îndia, estudo longitudinal com gestantes, lactantes e lactentes verificou que para cada 1 ano de aumento na idade da mãe a Concentração de Iodo Urinário (CIU) diminuía $5 \% 22$. Entretanto, no estudo longitudinal realizado na Etiópia, o risco de deficiência de iodo foi menor em gestantes com 35 a 49 anos de idade ${ }^{21}$.

Já a paridade e a idade gestacional quando maiores se associaram ao melhor estado nutricional de iodo à ingestão de alimentos fontes de sal iodado ${ }^{23-25}$. Porém alguns estudos não encontraram essa associação $0^{8,17,21}$.

O hábito de fumar na gestação e a lactação afeta negativamente o estado nutricional de iodo da mãe e do lactente ${ }^{24,25}$. O tabagismo materno pode provocar aborto espontâneo e prejudicar a capacidade da tireoide infantil de sintetizar hormônios. Em função disto, o recém-nascido pode nascer com transtornos mentais, além de reduzir em cerca de $50 \%$ o teor de iodo no leite materno, expondo a criança ao risco aumentado de deficiência desse nutriente ${ }^{26}$.

$\mathrm{Na}$ Catalunha, um estudo demostrou que as gestantes residentes nas montanhas apresentaram maior consumo de sal iodado e iodúria do que aquelas da área da costa ${ }^{27}$. Entretanto, estudo realizado no Irã, a CIU no primeiro e terceiro trimestre foi maior nas gestantes que vivem em regiões de planícies quando comparadas a regiões montanhosas ${ }^{28}$. As diferenças geográficas entre os países influenciam sobre a disponibilidade de iodo no solo e nos alimentos. No entanto, com a globalização do comércio de gêneros alimentícios, o consumo alimentar pouco difere entre as regiões litorâneas e montanhosas ${ }^{2}$.

O local de residência na zona rural das gestantes, crianças e lactantes em 19,2\% ( $\mathrm{n}=8)$ 
Quadro 1. Descrição dos estudos selecionados para a revisão sistemática.

\begin{tabular}{|c|c|c|c|c|}
\hline Referência & $\begin{array}{l}\text { Local / } \\
\text { Desenho do } \\
\text { Estudo }\end{array}$ & Público Alvo (n) & Hábito alimentar & Fatores Sociodemográficos \\
\hline $\begin{array}{l}\text { Schulze et } \\
\text { al. }^{8}(2003)\end{array}$ & $\begin{array}{l}\text { Nepal / } \\
\text { Transversal }\end{array}$ & $\begin{array}{l}\text { Gestantes (1021) } \\
\text { lactantes (1028) }\end{array}$ & - & $\begin{array}{l}\text { As mulheres apresentaram maior CIU na } \\
\text { gravidez comparado ao pós-parto. Não } \\
\text { houve relação entre idade materna, paridade, } \\
\text { idade gestacional e CIU. }\end{array}$ \\
\hline $\begin{array}{l}\text { Skeaff et } \\
\text { al. }^{29}(2005)\end{array}$ & $\begin{array}{l}\text { Nova } \\
\text { Zelândia / } \\
\text { Transversal }\end{array}$ & $\begin{array}{l}\text { Lactentes de } 6 \text { a } \\
24 \text { meses (230) }\end{array}$ & $\begin{array}{l}\text { CIU de crianças alimentadas } \\
\text { com a fórmula foi adequada. A } \\
\text { CIU de crianças em aleitamento } \\
\text { materno indicou deficiência } \\
\text { moderada. }\end{array}$ & $\begin{array}{l}\text { Não houve diferenças entre sexo, crianças } \\
\text { brancas e não-brancas e renda em relação a } \\
\text { CIU. }\end{array}$ \\
\hline \begin{tabular}{|l|} 
Semba et \\
al. $^{30}(2008)$
\end{tabular} & $\begin{array}{l}\text { Indonésia/ } \\
\text { Transversal }\end{array}$ & $\begin{array}{l}\text { Criança de } 0 \text { a } \\
59 \text { meses: favela } \\
\text { urbana (145.522) } \\
\text { e favela rural } \\
(445.546)\end{array}$ & $\begin{array}{l}\text { O consumo de sal não iodado } \\
\text { se associou ao baixo peso nas } \\
\text { crianças. }\end{array}$ & $\begin{array}{l}\text { As crianças pertencentes a famílias tanto } \\
\text { de favelas urbanas como rurais, eram mais } \\
\text { propensas a consumir sal iodado se os pais } \\
\text { tivessem maior escolaridade, idade e renda. }\end{array}$ \\
\hline $\begin{array}{l}\text { Costeira et } \\
\text { al. }{ }^{17}(2009)\end{array}$ & $\begin{array}{l}\text { Portugal/ } \\
\text { Longitudinal }\end{array}$ & Gestantes (140) & $\begin{array}{l}\text { Não houve relação entre o } \\
\text { consumo de peixe e CIU. O iodo } \\
\text { no leite materno estava abaixo } \\
\text { do valor recomendado. }\end{array}$ & $\begin{array}{l}\text { CIU foi inferior em gestantes com menor } \\
\text { renda. Não houve relação entre paridade e } \\
\text { idade com CIU. }\end{array}$ \\
\hline $\begin{array}{l}\text { Fisher et } \\
\text { al. } .^{31}(2011)\end{array}$ & $\begin{array}{l}\text { Vietnã / } \\
\text { Longitudinal }\end{array}$ & Gestantes (413) & - & $\begin{array}{l}\text { Paridade, escolaridade e ocupação foram } \\
\text { associadas ao uso de sal iodado. As gestantes } \\
\text { agricultoras e donas de casa eram mais } \\
\text { propensas a usar sal iodado. }\end{array}$ \\
\hline $\begin{array}{l}\text { Mackerras } \\
\text { et al. }{ }^{16} \\
(2011)\end{array}$ & $\begin{array}{l}\text { Austrália / } \\
\text { Longitudinal }\end{array}$ & $\begin{array}{l}\text { Gestantes (665) } \\
\text { Lactentes de } 0 \text { a } 6 \\
\text { meses (432) } \\
\text { Lactantes (467) }\end{array}$ & $\begin{array}{l}\text { O leite contribuiu para o } \\
\text { estado adequado de iodo. O } \\
\text { peixe fez apenas uma pequena } \\
\text { contribuição. }\end{array}$ & $\begin{array}{l}\text { Associação positiva entre o estado nutricional } \\
\text { de iodo adequado com o hábito de não } \\
\text { fumar, maior condição socioeconômica e } \\
\text { escolaridade das gestantes. }\end{array}$ \\
\hline $\begin{array}{l}\text { Menon et } \\
\text { al. }^{22} \\
(2011)\end{array}$ & $\begin{array}{l}\text { Índia/ } \\
\text { Longitudinal }\end{array}$ & $\begin{array}{l}\text { Gestantes }(220) \\
\text { Lactantes }(183) \\
\text { Lactentes }(180)\end{array}$ & - & $\begin{array}{l}\text { Idade materna, duração da gestação, } \\
\text { concentração de iodo salino, escolaridade } \\
\text { materna }<8 \text { anos e a localização rural } \\
\text { associou-se à CIU. }\end{array}$ \\
\hline $\begin{array}{l}\text { Vila et al. }{ }^{27} \\
(2011)\end{array}$ & $\begin{array}{l}\text { Espanha/ } \\
\text { Longitudinal }\end{array}$ & Gestantes (267) & $\begin{array}{l}\text { A suplementação com iodo } \\
\text { foi maior na área da costa. } \mathrm{O} \\
\text { consumo de peixe se associou a } \\
\text { CIU adequada. }\end{array}$ & $\begin{array}{l}\text { As gestantes residentes nas montanhas } \\
\text { apresentaram maiores CIU e consumo de sal } \\
\text { iodado do que aquelas da área da costa. }\end{array}$ \\
\hline $\begin{array}{l}\text { Garcia- } \\
\text { Garcia et } \\
\text { al. }{ }^{32} \\
(2012)\end{array}$ & $\begin{array}{l}\text { Espanha/ } \\
\text { Transversal }\end{array}$ & $\begin{array}{l}1387 \text { crianças } \\
1 \text { e } 6 \text { anos }(478) \\
6 \text { e } 12 \text { anos }(505) \\
12 \text { e } 16(404)\end{array}$ & $\begin{array}{l}\text { Produtos lácteos e os vegetais } \\
\text { foram protetores para o estado } \\
\text { nutricional de iodo adequado. }\end{array}$ & $\begin{array}{l}\text { A prevalência deficiência de iodo foi menor } \\
\text { no grupo de } 1 \text { a } 6 \text { anos. } 47,3 \% \text { das famílias } \\
\text { com menor renda não utilizavam sal iodado. }\end{array}$ \\
\hline $\begin{array}{l}\text { Macedo et } \\
\text { al. }^{33} \\
(2012)\end{array}$ & $\begin{array}{l}\text { Brasil/ } \\
\text { Transversal }\end{array}$ & $\begin{array}{l}\text { Criança de } 6 \text { a } 71 \\
\text { meses (337) }\end{array}$ & $\begin{array}{l}\text { Local de armazenamento do } \\
\text { sal iodado, uso de tempero } \\
\text { industrial e origem da água de } \\
\text { consumo se associaram a CIU. }\end{array}$ & $\begin{array}{l}\text { Crianças da zona rural tiveram valores de } \\
\text { CIU três vezes menor }(27 \% \text { e } 9,4 \%) \text { quando } \\
\text { comparadas às urbanas. Crianças com menor } \\
\text { renda familiar foram deficientes de iodo. }\end{array}$ \\
\hline $\begin{array}{l}\text { Rostami et } \\
\text { al. } .^{28} \\
(2012)\end{array}$ & $\begin{array}{l}\text { Irã/ } \\
\text { Transversal }\end{array}$ & Gestantes (489) & - & $\begin{array}{l}\text { CIU no primeiro e terceiro trimestre foi } \\
\text { maior nas gestantes que vivem em regiões de } \\
\text { planícies comparada a região montanhosa. }\end{array}$ \\
\hline $\begin{array}{l}\text { Shamim et } \\
\text { al. }{ }^{18}(2012)\end{array}$ & $\begin{array}{l}\text { Bangladesh / } \\
\text { Transversal }\end{array}$ & $\begin{array}{l}\text { Gestantes } \\
\leq 16 \text { semanas } \\
(1.376) \text { e } \\
\geq 32 \text { semanas } \\
(1.114)\end{array}$ & - & $\begin{array}{l}\text { Gestantes casadas com homens que tinham } \\
\text { ao menos o ensino médio, pertencentes a } \\
\text { famílias com melhor renda, com eletricidade } \\
\text { e televisão eram mais propensas a consumir } \\
\text { sal iodado. }\end{array}$ \\
\hline $\begin{array}{l}\text { Brantsæter } \\
\text { et al. } .^{24} \\
(2013)\end{array}$ & $\begin{array}{l}\text { Noruegua / } \\
\text { Longitudinal }\end{array}$ & $\begin{array}{l}\text { Gestantes } \\
(61.904) \\
\text { Lactentes } \\
(108.000) \\
\end{array}$ & $\begin{array}{l}\text { O consumo de leite, iogurte, frutos } \\
\text { do mar, ovos e suplemento de } \\
\text { iodo contribuíram para o estado } \\
\text { nutricional de iodo adequado. }\end{array}$ & $\begin{array}{l}\text { A ingestão de alimentos fonte de iodo não } \\
\text { diferiu em relação à idade materna, educação, } \\
\text { uso de suplementos ou estado civil. }\end{array}$ \\
\hline
\end{tabular}


Quadro 1. Descrição dos estudos selecionados para a revisão sistemática (Continuação).

\begin{tabular}{|c|c|c|c|c|}
\hline Referência & $\begin{array}{l}\text { Local / } \\
\text { Desenho do } \\
\text { Estudo }\end{array}$ & Público Alvo (n) & Hábito alimentar & Fatores Sociodemográficos \\
\hline $\begin{array}{l}\text { Habimana } \\
\text { et al. } \\
(2013)\end{array}$ & \begin{tabular}{|l|} 
República \\
Democrática \\
do Congo/ \\
Transversal \\
\end{tabular} & $\begin{array}{l}\text { Gestantes }(225) \\
\text { Lactantes }(75) \\
\text { Grupo controle } \\
(75) \\
\end{array}$ & - & $\begin{array}{l}\text { Na área urbana a mediana de CIU foi } \\
\text { adequada, na área semiurbana levemente } \\
\text { deficiente e na área rural moderadamente } \\
\text { deficiente. }\end{array}$ \\
\hline $\begin{array}{l}\text { Lean et al. }{ }^{19} \\
(2014)\end{array}$ & $\begin{array}{l}\text { Índia/ } \\
\text { Longitudinal }\end{array}$ & $\begin{array}{l}\text { Gestantes } \\
17 \text { semanas }(132) \\
34 \text { semanas }(151) \\
\text { Lactentes }(283)\end{array}$ & $\begin{array}{l}\text { O consumo de produtos lácteos } \\
\text { foi maior para as gestantes com } \\
\text { CIU no quartil mais alto na } 34^{\circ} \\
\text { semana }\end{array}$ & $\begin{array}{l}\text { A adequada CIU se associou a maior renda } \\
\text { e escolaridade das gestantes na } 34^{\circ} \text { semanas. } \\
\text { Não houve associação entre a localização e } \\
\text { CIU. }\end{array}$ \\
\hline $\begin{array}{l}\text { Bath et al. }{ }^{23} \\
(2014)\end{array}$ & $\begin{array}{l}\text { Reino Unido/ } \\
\text { Transversal }\end{array}$ & Gestantes (100) & $\begin{array}{l}\text { O consumo de frutos do mar, } \\
\text { leite, queijo, ovo e suplementos } \\
\text { contribuiu para a adequação do } \\
\text { estado nutricional de iodo. }\end{array}$ & $\begin{array}{l}\text { A excreção urinária de iodo foi maior em } \\
\text { gestantes com mais de } 35 \text { anos, mas ao } \\
\text { examinar a relação entre idade materna } \\
\text { avançada e melhor estado de iodo, observou- } \\
\text { se correlação negativa. }\end{array}$ \\
\hline $\begin{array}{l}\text { Ferreira et } \\
\text { al. } .^{25}(2014)\end{array}$ & $\begin{array}{l}\text { Brasil / } \\
\text { Transversal }\end{array}$ & $\begin{array}{l}\text { Gestantes }(191) \\
\text { Grupo controle } \\
(58)\end{array}$ & - & $\begin{array}{l}\text { Aborto e o hábito de fumar se associaram a } \\
\text { menor CIU. Já a idade gestacional avançada } \\
\text { se associou a melhor CIU. }\end{array}$ \\
\hline $\begin{array}{l}\text { Kedir et } \\
\text { al. }{ }^{21}(2014)\end{array}$ & $\begin{array}{l}\text { Etiópia/ } \\
\text { Longitudinal }\end{array}$ & Gestantes (435) & $\begin{array}{l}\text { O consumo de leite três vezes } \\
\text { ao mês, repolho uma vez } \\
\text { por semana e o sal iodado } \\
\text { diariamente exerceu papel } \\
\text { protetor contra a deficiência de } \\
\text { iodo em gestantes. }\end{array}$ & $\begin{array}{l}\text { O risco de deficiência de iodo aumentou } \\
3 \text { vezes em gestantes analfabetas e que } \\
\text { pertenciam a famílias com } 5 \text { membros ou } \\
\text { mais. O risco de deficiência de iodo foi } \\
\text { menor em multíparas e em gestantes com } 35 \\
\text { a } 49 \text { anos de idade. }\end{array}$ \\
\hline $\begin{array}{l}\text { Bath et al. }{ }^{20} \\
(2015)\end{array}$ & $\begin{array}{l}\text { Reino Unido / } \\
\text { Longitudinal }\end{array}$ & $\begin{array}{l}\text { Gestantes na } 12 \\
\text { semanas (228) } \\
\text { na } 20(222) \text { e na } \\
35 \text { semana }(212)\end{array}$ & $\begin{array}{l}\text { CIU foi maior nas gestantes } \\
\text { que relataram consumir uma } \\
\text { porção de produtos lácteos/dia e } \\
\text { castanha do Brasil. }\end{array}$ & $\begin{array}{l}\text { Não houve associação entre etnia, classe } \\
\text { social e uso de tabaco com a relação } \\
\text { iodo-creatinina das gestantes. Maior } \\
\text { idade materna e renda foram associados } \\
\text { positivamente à relação iodo-creatinina. }\end{array}$ \\
\hline $\begin{array}{l}\text { Kumar; } \\
\text { Berkman }^{15} \\
(2015)\end{array}$ & $\begin{array}{l}548 \text { distritos da } \\
\text { India/ } \\
\text { Transversal }\end{array}$ & $\begin{array}{l}\text { Crianças de } \\
0 \text { a } 71 \text { meses } \\
(287.247)\end{array}$ & $\begin{array}{l}\text { Houve associação entre o } \\
\text { consumo de sal não iodado com } \\
\text { baixo peso nas crianças. }\end{array}$ & $\begin{array}{l}\text { Cerca de } 45,8 \% \text { das crianças de baixa } \\
\text { renda não usaram sal iodado. } O R \text { para } \\
\text { famílias de baixa renda foi de } 2,55 \text {. } \\
\text { Crianças pertencentes a famílias rurais, sem } \\
\text { eletricidade, instalações sanitárias e que o } \\
\text { chefe tinha nível de educação inferior foram } \\
\text { menos propensas a usar sal iodado. }\end{array}$ \\
\hline $\begin{array}{l}\text { Anaforoglu } \\
\text { et al. }{ }^{35}(2016)\end{array}$ & \begin{tabular}{|l|} 
Turquia/ \\
Transversal \\
\end{tabular} & Gestantes (864) & - & $\begin{array}{l}\text { CIU não diferiu quanto ao nível de } \\
\text { escolaridade, paridade e renda. }\end{array}$ \\
\hline $\begin{array}{l}\text { Jorgensen } \\
\text { et al. }{ }^{7} \\
(2016)\end{array}$ & $\begin{array}{l}\text { Austrália/ } \\
\text { Longitudinal }\end{array}$ & Lactantes (30) & $\begin{array}{l}\text { A ingestão diária de leite de vaca } \\
\text { associou-se à maiores valores na } \\
\text { concentração de iodo no leite } \\
\text { materno. }\end{array}$ & $\begin{array}{l}\text { A baixa renda das famílias das lactantes } \\
\text { e a menor escolaridade se associou } \\
\text { negativamente ao uso de suplemento de } \\
\text { iodo. }\end{array}$ \\
\hline $\begin{array}{l}\text { Kramer et } \\
\text { al. }{ }^{11}(2016)\end{array}$ & $\begin{array}{l}46 \text { países de } \\
\text { diferentes na- } \\
\text { cionalidades / } \\
\text { Transversal }\end{array}$ & $\begin{array}{l}\text { Crianças entre } \\
0 \text { e } 59 \text { meses } \\
(655.811)\end{array}$ & - & $\begin{array}{l}\text { Crianças pertencentes a famílias que não } \\
\text { faziam uso de sal iodado foram menos } \\
\text { propensas a ser imunizadas, mais propensas } \\
\text { a viver em áreas rurais e em famílias de baixa } \\
\text { renda. E as mães eram mais propensas a se } \\
\text { casar, de não ter educação primária e vínculo } \\
\text { empregatício. }\end{array}$ \\
\hline $\begin{array}{l}\text { Nazeri et } \\
\text { al. }{ }^{36}(2016)\end{array}$ & $\begin{array}{l}\text { Irã / } \\
\text { Transversal }\end{array}$ & $\begin{array}{l}\text { Lactantes }(147) \\
\text { e seus recém- } \\
\text { nascidos }\end{array}$ & - & $\begin{array}{l}\text { CIU foi adequada em mães com maior } \\
\text { escolaridade, paridade e com vínculo } \\
\text { empregatício. CIU foi maior em lactentes } \\
\text { com maior peso ao nascer e do sexo } \\
\text { feminino. }\end{array}$ \\
\hline
\end{tabular}


Quadro 1. Descrição dos estudos selecionados para a revisão sistemática.

\begin{tabular}{|l|l|l|l|l|}
\hline Referência & $\begin{array}{c}\text { Local / } \\
\text { Desenho do } \\
\text { Estudo }\end{array}$ & Público Alvo (n) & Hábito alimentar & Fatores Sociodemográficos \\
\hline $\begin{array}{l}\text { Torres et } \\
\text { al. }{ }^{9}(2017)\end{array}$ & $\begin{array}{l}\text { Espanha/ } \\
\text { Transversal }\end{array}$ & Gestantes (985) & $\begin{array}{l}\text { A ingestão de um copo de leite } \\
\text { teve efeito protetor que foi ainda } \\
\text { maior se associado ao consumo } \\
\text { de sal iodado. }\end{array}$ & $\begin{array}{l}\text { CIU média não diferiu de acordo com a } \\
\text { escolaridade, local de residência rural ou } \\
\text { urbano e uso de tabaco nas gestantes. }\end{array}$ \\
\hline $\begin{array}{l}\text { Wang et } \\
\text { al. }^{10}(2017)\end{array}$ & $\begin{array}{l}\text { China/ } \\
\text { Transversal }\end{array}$ & Gestantes (8159) & $\begin{array}{l}\text { As gestantes da zona rural e da } \\
\text { costa que consomem sal iodado } \\
\text { tiveram maior CIU. }\end{array}$ & $\begin{array}{l}\text { As gestantes da zona rural tiveram maior } \\
\text { CIU quando comparadas as urbanas. }\end{array}$ \\
\hline
\end{tabular}

${ }^{*} \mathrm{CIU}=$ Concentração de Iodo Urinário.

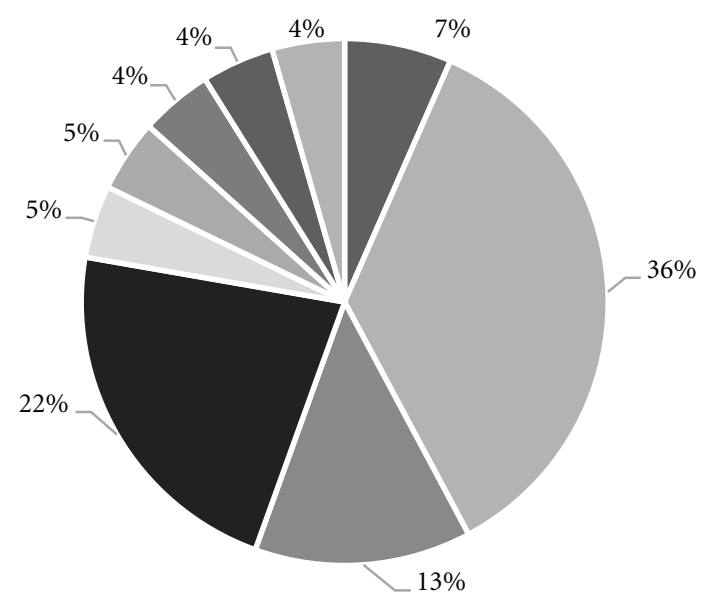
Sal Iodado
Produtos Lácteos
Suplementos de Iodo
Peixes
Castanha
Repolho
Ovo
Fórmula Infantil
Vegetal

Gráfico 1. Alimentos identificados nos estudos que se relacionaram ao estado de iodo.

dos estudos demostrou relação negativa com o estado nutricional de iodo. Em estudo realizado no Brasil, as crianças residentes na zona rural tiveram valores de CIU três vezes inferiores $(27 \%$ e 9,4\%) quando comparadas às urbanas ${ }^{33}$. Já na República Democrática do Congo, a CIU de gestantes e lactantes na área urbana foi adequada, na semiurbana levemente deficiente e na zona rural moderadamente deficiente ${ }^{34}$. Entretanto, em um estudo realizado na China, a CIU das gestantes da zona rural foi maior quando comparada às urbanas $^{10}$. Já em Barcelona, a CIU não diferiu de acordo com o local de residência rural ou urbano das gestantes 9.

Na zona rural, o consumo de sal iodado é menor, visto que as mulheres, em sua maioria, tendem a ser menos alfabetizadas e a não possuir qualificação profissional ${ }^{18}$. Entretanto, no estudo realizado no Vietnã, as gestantes agricultoras e donas de casa eram mais propensas a usar sal iodado na culinária. Segundo os autores, as mulheres com outras ocupações não utilizavam sal iodado por falta de familiarização com o produto, utilização de temperos naturais, mudanças no 
paladar (relataram sabor amargo do sal iodado) e indisponibilidade para a compra local ${ }^{31}$.

Estudo realizado em 46 países, as crianças pertencentes a famílias que não faziam uso de sal iodado foram menos propensas a ser imunizadas, mais propensas a viver em áreas rurais e em famílias de baixa renda. E as mães eram mais propensas a se casar, de não ter educação primária e vínculo empregatício ${ }^{11}$. Esses dados transparecem o cenário de insegurança alimentar e reforçam que os fatores sociodemográficos são determinantes para a nutrição adequada de iodo.

O estado de iodo é variável em todos os trimestres de gestação e a alimentação é crucial para evitar a deficiência na mãe e no recém-nascido. $\mathrm{Na}$ Índia, um estudo com 287.247 crianças verificou associação entre o consumo de sal não iodado e o baixo peso nas crianças, o mesmo resultado foi observado nas crianças de favelas urbanas e rurais $^{15,31}$.

Em estudo transversal realizado com 655.811 crianças de diferentes nacionalidades, a utilização de sal iodado se associou positivamente ao estado nutricional de iodo, porém quando estava indisponível para o consumo das gestantes, as crianças tinham $15,7 \%$ maior probabilidade de nascer com baixo peso. Porém, o crescimento ponderal foi maior nas crianças que consumiam sal não iodado ${ }^{11}$. No entanto, é preciso cautela na análise desses resultados, pois o baixo peso pode ter causa multifatorial, com complexa interação entre fatores nutricionais, ambientais, psicossociais e socioeconômicos, sendo o sal iodado apenas mais um fator.

Para a correta utilização do sal iodado, é preciso considerar a sua forma de armazenamento, assim como a adição no processo de cozimento, que deve ser no final, uma vez que quando o alimento é aquecido a temperaturas elevadas, pode ocorrer até $70 \%$ de perda do conteúdo de iodo ${ }^{31}$.

O consumo de alimentos fontes de iodo e a suplementação exerceram papel protetor contra a deficiência. O consumo de produtos lácteos em $36 \%(n=8)$ dos estudos se associou a CIU adequada, em que a ingestão de um copo de leite por dia teve efeito protetor que foi ainda maior se associado ao consumo de sal iodado, efeito semelhante ao da suplementação9 . O iodo é armazenado na tireoide e se a ingestão dietética for diária, a nutrição iódica adequada é garantida ${ }^{19}$.

A CIU dos lactentes em uso de fórmula infantil foi maior quando comparada aos de aleitamento materno em Portugal e na Nova Zelândia. Destaca-se que o iodo presente no leite materno é melhor absorvido, no entanto, ele reflete a alimentação e o estado nutricional de iodo da mãe, o que justifica a variação. Com a alimentação complementar acredita-se que o estado nutricional de iodo dos lactentes em aleitamento materno melhore, uma vez que outros alimentos fontes serão ingeridos ${ }^{29,37}$.

O ponto forte deste estudo é que todos os fatores sociodemográficos foram abordados, permitindo um panorama geral de como estes podem afetar o estado nutricional de iodo do grupo materno infantil, permitindo o delineamento de estratégias efetivas para controle da deficiência desse nutriente no grupo materno-infantil. Com relação às limitações: foram incluídos artigos de diferentes delineamentos, por isso identificou-se resultados heterogêneos, dificultando a comparação. E apenas um estudo avaliou o estado nutricional iódico de lactentes em uso de fórmula infantil e em aleitamento materno exclusivo. Logo, é preciso que mais estudos sejam realizados a fim de elucidar e esclarecer essa associação.

Portanto, os fatores sociodemográficos assim como os diferentes hábitos alimentares podem estar relacionados ao estado nutricional de iodo durante toda a gestação, a lactação e o desenvolvimento infantil. Por isso, é preciso criar estratégias mais específicas e efetivas, para assim evitar as desordens provocadas pela deficiência de iodo. 


\section{Colaboradores}

AC Candido foi responsável pela concepção, delineamento, interpretação dos dados e redação do artigo. As autoras SE Priore, MS Macedo e SCC Franceschini revisaram criticamente o artigo. Todos as autoras aprovaram a versão final do artigo publicada.

\section{Agradecimentos}

O presente trabalho foi realizado com apoio da Coordenação de Aperfeiçoamento de Pessoal de Nível Superior - Brasil (CAPES) - Código de Financiamento 001; ao Conselho Nacional de Desenvolvimento Científico e Tecnológico (CNPq) e à Fundação de Amparo e Pesquisa do Estado de Minas Gerais (FAPEMIG).

\section{Referências}

1. Bath SC, Rayman MP. Europe PMC Funders Group Europe PMC Funders Author Manuscripts A review of the iodine status of UK pregnant women and its implications for the offspring. 2016; 37(4):619-629.

2. World Health Organization (WHO). Assessment of the iodine deficiency disorders and monitoring their elimination. Geneva: WHO; 2007.

3. Haldimann M, Alt A, Blanc A, Blondeau K. Iodine content of food groups. J Food Compos Anal 2005; 18(6):461-471.

4. Berbel P, Mestre JL, Santamaría A, Palazón I, Franco A, Graells M, González-Torga A, Escobar GM. Delayed Neurobehavioral Development in Children Born to Pregnant Women with Mild Hypothyroxinemia During the First Month of Gestation: The Importance of Early Iodine Supplementation. Thyroid 2009; 19(5):511-519.

5. Glinoer D. The importance of iodine nutrition during pregnancy. Public Health Nutr 2007; 10(12A):15421546.

6. Liberati A, Altman DG, Tetzlaff J, Mulrow C, Gøtzsche PC, Ioannidis JPA, Clarke M, Devereaux PJ, Kleijnen J, Moher D. The PRISMA statement for reporting systematic reviews and meta-analyses of studies that evaluate health care interventions: Explanation and elaboration. PLoS Med 2009; 6:7.

7. Jorgensen A, O'Leary P, James I, Skeaff S, Sherriff J. Assessment of breast milk iodine concentrations in lactating women in western Australia. Nutrients 2016; 8(11):1-8.

8. Schulze KJ, West Junior KP, Gautschi LA, Dreyfuss ML, LeClerq SC, Dahal BR, Wu LS-F, Khatry SK. Seasonality in urinary and household salt iodine content among pregnant and lactating women of the plains of Nepal. Eur J Clin Nutr 2003; 57(8):969-976.

9. Torres MT, Francés L, Vila L, Manresa JM, Falguera G, Prieto G, Casamitjana R, Toran P \& the IODEGEST study group. Iodine nutritional status of women in their first trimester of pregnancy in Catalonia. $B M C$ Pregnancy Childbirth 2017; 17(1):249.

10. Wang Z, Zhu W, Mo Z, Wang Y, Mao G, Wang X, Lou $X$. An Increase in Consuming Adequately Iodized Salt May Not Be Enough to Rectify Iodine Deficiency in Pregnancy in an Iodine-Sufficient Area of China. Int J Environ Res Public Health [Internet]. 20 de fevereiro de 2017 [acessado 2017 Nov 14]; 14(2):206. Available at: http://www.mdpi.com/1660-4601/14/2/206

11. Krämer M, Kupka R, Subramanian SV, Vollmer S. Association between household unavailability of iodized salt and child growth: Evidence from 89 demographic and health surveys. Am J Clin Nutr 2016; 104(4):10931100.

12. Katz PM, Leung AM, Braverman LE, Pearce EM, Tomlinson G, He X, Vertes BS, Okun N, Walfish PG, Feig DS. Iodine Nutrition During Pregnancy In Toronto, Canada. Endocr Pract 2013; 19(2):206-211.

13. Andersson M, Karumbunathan V, Zimmermann MB. Global Iodine Status in 2011 and Trends over the Past Decade. J Nutr 2012; 142(4):744-750.

14. Zou Y, Lou X, Ding G, Mo Z, Zhu W, Mao G. A cross-sectional comparison study on the iodine nutritional status between rural and urban residents in Zhejiang Province, China. BMJ Open 2014; 4(6):e005484-e005484. 
15. Kumar S, Berkman LF. Association of inadequately iodized salt use with underweight among young children in India. Asia-Pacific J Public Health 2015; 27(2):185-194.

16. Mackerras D, Powers J, Boorman J, Loxton D, Giles GG. Estimating the impact of mandatory fortification of bread with iodine on pregnant and post-partum women. J Epidemiol Community Health 2011; 65(12):1118-1122.

17. Costeira MJ, Oliveira P, Ares S, Escobar GM, Palha JA. Iodine status of pregnant women and their progeny in the Minho Region of Portugal. Thyroid. 2009; 19(2):157-163.

18. Shamim A, Christian P, Schulze KJ, Ali H, Kabir A, Rashid M, Labrique A, Salamatullah Q, West Junior KP. Iodine status in pregnancy and household salt iodine content in rural Bangladesh. Matern Child Nutr 2012; 8(2):162-173.

19. Lean MI, Lean MEJ, Yajnik CS, Bhat DS, Joshi SM, Raut DA, Lubree HG, Combet E. Iodine status during pregnancy in India and related neonatal and infant outcomes. Public Health Nutr 2014; 17(6):1353-1362.

20. Bath SC, Furmidge-Owen VL, Redman CW, Rayman MP. Gestational changes in iodine status in a cohort study of pregnant women from the United Kingdom. Am J Clin Nutr 2015;101(6):1180-1187.

21. Kedir H, Berhane Y, Worku A. Subclinical Iodine Deficiency among Pregnant Women in Haramaya District, Eastern Ethiopia: A Community-Based Study. J Nutr Metab 2014; 2014:878926.

22. Menon KC, Skeaff SA, Thomson CD, Gray AR, Ferguson EL, Zodpey S, Saraf A, Das PK, Pandav CS. The Effect of Maternal Iodine Status on Infant Outcomes in an Iodine-Deficient Indian Population. Thyroid 2011; 21(12):1373-1380.

23. Bath SC, Walter A, Taylor A, Wright J, Rayman MP. Iodine deficiency in pregnant women living in the South East of the UK: the influence of diet and nutritional supplements on iodine status. Br J Nutr 2014; 111(9):1622-1631.

24. Brantsæter AL, Abel MH, Haugen M, Meltzer HM. Risk of suboptimal iodine intake in pregnant norwegian women. Nutrients 2013; 5(2):424-440.

25. Ferreira SMS, Navarro AM, Magalhães PKR, Maciel LMZ. Iodine insufficiency in pregnant women from the State of São Paulo. Arq Bras Endocrinol Metabol 2014; 58(3):282-287.

26. Laurberg P, Nøhr SB, Pedersen KM, Fuglsang E. Iodine Nutrition in Breast-Fed Infants is Impaired by Maternal Smoking. J Clin Endocrinol Metab 2004; 89(1):181-187.

27. Vila L, Serra-Prat M, Castro A, Palomera E, Casamitjana R, Legaz G, Barrionuevo C, Muñoz JA, García AJ, Lal-Trehan S, García A, Durán J, Puig-Domingo M. Iodine nutritional status in pregnant women of two historically different iodine-deficient areas of Catalonia, Spain. Nutrition 2011; 27(10):1029-1033.

28. Rostami R, Beiranvend A, Nourooz-Zadeh J. Nutritional iodine status in gestation and its relation to geographic features in Urmia County of northwest Iran. Food Nutr Bull 2012; 33(4):267-272.
29. Skeaff SA, Ferguson EL, McKenzie JE, Valeix P, Gibson RS, Thomson CD. Are breast-fed infants and toddlers in New Zealand at risk of iodine deficiency? Nutrition 2005; 21(3):325-331.

30. Semba RD, de Pee S, Hess SY, Sun K, Sari M, Bloem MW. Child malnutrition and mortality among families not utilizing adequately iodized salt in Indonesia. Am J Clin Nutr 2008; 87(2):438-444.

31. Fisher J, Tran T, Biggs B, Tran T, Dwyer T, Casey G, Tho DH, Hetze B. Statut iodé enfin de grossesse et déterminants psychosociaux de l'utilisation de sel iodé dans les régions rurales du nord du Viet Nam. Bull World Health Organ 2011; 89(11):813-820.

32. Garcia-Garcia E, Vázquez-López MA, García-Fuentes E, Rodríguez-Sánchez FI, Muñoz FJ, Bonillo-Perales A, Soriguer F. Iodine intake and prevalence of thyroid autoimmunity and autoimmune thyroiditis in children and adolescents aged between 1 and 16 years. Eur J Endocrinol 2012; 167(3):387-392.

33. Macedo MS, Teixeira RA, Bonomo E, Silva CAM, Silva ME, Sakurai E, Carneiro M, Lamounier JA. Deficiência de iodo e fatores associados em lactentes e pré-escolares de um município do semiárido de Minas Gerais, Brasil, 2008. Cad Saude Publica 2012; 28(2):346-356.

34. Habimana L, Twite KE, Wallemacq P, De Nayer P, Daumerie C, Donnen P, Kalenga MK, Robert A. Iodine and iron status of pregnant women in Lubumbashi, Democratic Republic of Congo. Public Health Nutr 2013; 16(8):1362-1370.

35. Anaforoğlu İ, Algün E, İnceçayır Ö, Topbaş M, Erdoğan MF. Iodine status among pregnant women after mandatory salt iodisation. Br J Nutr 2016; 115(3):405-410.

36. Nazeri P, Mirmiran P, Hedayati M, Mehrabi Y, Delshad H, Azizi F. Can postpartum maternal urinary iodine be used to estimate iodine nutrition status of newborns? Br J Nutr 2016; 115(7):1226-1231.

37. New Zealand. Ministry of Health. Guidelines for Healthy Infants and Toddlers (Aged 0-2) A background paper. Wellington: Ministry of Health; 2012.

Artigo apresentado em 01/09/2018

Aprovado em 19/06/2019

Versão final apresentada em 21/06/2019

Editores-chefes: Romeu Gomes, Antônio Augusto Moura da Silva 